\title{
Design and Implementation Issues of Clustering in Wireless Sensor Networks
}

\author{
K. Pradeepa \\ Department of Computer \\ Applications. \\ Sri Krishna College of \\ Engineering \& Technology \\ Coimbatore
}

\author{
W. Regis Anne \\ Department of Computer \\ Applications. \\ Sri Krishna College of \\ Engineering \& Technology \\ Coimbatore
}

\author{
S. Duraisamy \\ Department of Computer \\ Applications. \\ Sri Krishna College of \\ Engineering \& Technology \\ Coimbatore
}

\begin{abstract}
Sensors have become a very trendy research area during the last few years covering a wide range of applications such as habitat monitoring, military surveillance, information collecting etc...Sensors used for these purposes needs to be deployed very densely and in a random fashion. They should be able to operate without human intervention. Clustering is a technique employed to increase the various capabilities of a sensor network. In this paper we discuss about the design and implementation issues of clustering algorithms employed in sensor networks.
\end{abstract}

\section{General Terms}

Clustering in Wireless Sensor Network, Design Issues in Clustering, and Implementation Issues in Clustering.

\section{Keywords}

Clustering, Flat Network, Hierarchical Network Sensor Networks, Overlapping.

\section{INTRODUCTION}

Recent development in the field of miniaturization led to the development of small tiny sensor nodes which can be deployed on various locations for achieving certain goals. The sensors are capable of self organizing themselves to form a network. Sensors are randomly deployed in the area of interest. As the devices are very small and inexpensive, they can be deployed in large numbers for better accurate sensing. They monitor conditions at different locations such as temperature, humidity, pressure, vehicular movement, soil makeup, lightning condition, noise levels, the presence or absence of certain kind of object etc. The most straight forward application of sensor technology is to monitor remote locations without human interruption [1-3]. Applications include military, home, Habitat Monitoring, Agriculture etc.For a vast area to be sensed, it is foreseen that even thousands of thousands of sensor nodes are used due to the possibility of damage during deployment. Operating such a huge and complex network requires scalable architecture and management strategies. Scalability in a sensor network can be achieved by a technique known as Clustering [4] where the sensor nodes are grouped in to various clusters and each cluster having a cluster head $(\mathrm{CH})$ [4].The cluster heads may be predesigned by the network designer or elected by the sensors in the network.

Clustering algorithms for sensor networks improves network scalability by handling two important problems regarding the size and mobility of the network. Various clustering algorithms have been proposed by the research community. They vary according to the overall network architecture, Node deploying methods and based on the characteristics of the $\mathrm{CH}$ node. Number of cluster member will vary according to the application. Usually a cluster head is a node which is very rich in energy resources.

Sometimes cluster heads may form a second tier in the network. $\mathrm{CH}$ in such architecture acts as relay nodes [5]. Relay nodes have been proposed in sensor networks for achieving various objectives like data gathering, reduction of transmission range, fault tolerance etc. the communication from the relay nodes and base stations can either be singlehop or multi-hop.[5]. In a single-hop model $\mathrm{CHs}$ send their data directly to the base station. Where as in multi-hop $\mathrm{CHs}$ send their data through other $\mathrm{CHs}$, thus $\mathrm{CHs}$ in such models not only acts as relay nodes for transferring the data to base station, but also collects the data from other relay nodes and transfer the data to the base station.[5]

In this paper we have presented various design issues and challenges that occur with clustering in wireless sensor networks. In Section1 we have given an introduction regarding sensor networks, Section 2 discusses the decision metrics for clustering, Section 3 describes the architecture of a flat sensor network without clustering, drawbacks of the flat network, architecture of a hierarchical networks and its advantages, and Section 4 discusses the various design and implementation issues of clustering in sensor network and in Section 5 we have simulated various clustering schemes in sensor networks. We have analyzed how various types of clustering schemes affect the network performance and its lifetime. Variations in energy consumption of the sensor nodes depending on the clustering schemes is also been depicted by various energy consumption graphs.

\section{DECISION METRICS}

The decision of a sensor node to become $\mathrm{CH}$ or to join an existing cluster is typically based on the following metrics.

\subsection{Time}

A node may become a $\mathrm{CH}$ on a time dependable basis. For example a $\mathrm{CH}$ may be the first node in its neighborhood that declares itself as $\mathrm{CH}$.

\subsection{Probability}

A node may become a $\mathrm{CH}$ depending on a probabilistic measure. The probability may depend on the number of nodes in the network, global aggregate energy, local residual energy, number of times the nodes has been a $\mathrm{CH}$, cluster size etc. 


\subsection{Weight}

A weight is an application specific number assigned to every node in the network. The weight may depend on multiple measures such as the node degree. Distance to neighbours, movement speed, energy left capabilities. The node ID is used to break ties. A node in the sensor network may become a $\mathrm{CH}$ if it has the highest weight among a group of nodes, depending on the decision range. Similarly a node may choose to join the cluster head with the highest weight.

\subsection{Semantics}

Semantic properties refer to the relationship between pairs of nodes or among nodes in a group. Semantic properties include distance between nodes, availability paths between nodes, similar or relative mobility, and location attribute or event type detected. Clusters may be formed based on similar semantic properties of nodes.

The decision process may depend on more than one of the above metrics. For example the $\mathrm{CH}$ may be probabilistically selected, but the ordinary node chooses a $\mathrm{CH}$ based on a semantic property, (e.g. the minimum distance to the neighboring cluster heads). Similarly nodes are grouped based on the semantic information and the $\mathrm{CH}$ is chosen depending on the weight.

\section{ARCHITECTURE OF CLUSTERING}

A flat network is a collection of homogeneous sensor node. All nodes transmit their data either directly or via the nodes that lie between the transmitting nodes and the $\mathrm{CH}$. Architecture of a flat network is shown in the Fig 1. The main drawback of this architecture is since all the nodes communicate directly with the $\mathrm{CH}$ very soon they will lose their energy. Tasks like collection and dissemination of data will become tougher.

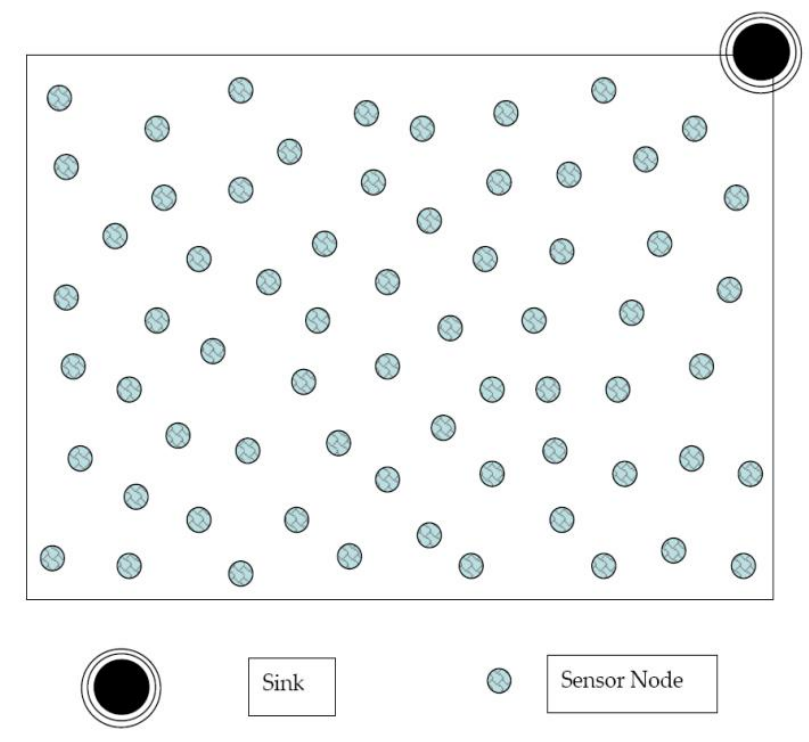

Fig 1: Architecture of a Flat Network

Another important drawback is in a flat network it is very difficult to add extra nodes to the existing network. So scalability is tougher in a flat type of sensor network. Flat networks are progressively replaced with hierarchical networks. If nodes are directly managed by the $\mathrm{CH}$ it would result in management delay, communication overhead and management complexity. This in turn could lead to a less responsive and less energy efficient network. It is well known that traffic in a sensor network can be minimized by aggregating similar data from physically adjacent sensors using cluster head approach in HWSN's. A node in the cluster is nominated as $\mathrm{CH}$ which is then in charge of collecting, aggregating and transmitting the data from all nodes within its cluster to the $\mathrm{CH}$. Since the majority of the nodes need to communicate to a shorter distance, their energy is conserved to a great extent. Clustering provides a efficient framework for routing information, local data aggregation, distributed management control and local decision making.

The $\mathrm{CH}$ needs to perform additional tasks of aggregating and transmitting the data from the nodes in the cluster to the $\mathrm{CH}$ and hence consume more energy. One way of conserving the energy of the $\mathrm{CH}$ is by rotating the role of $\mathrm{CH}$ between different nodes. Three main phases of clustering are as follows
i) Cluster Initialization
ii) Cluster Formation
iii) Cluster Maintenance

Fig 2 describes the hierarchy of nodes in a clustered network. The advantages of this setup includes

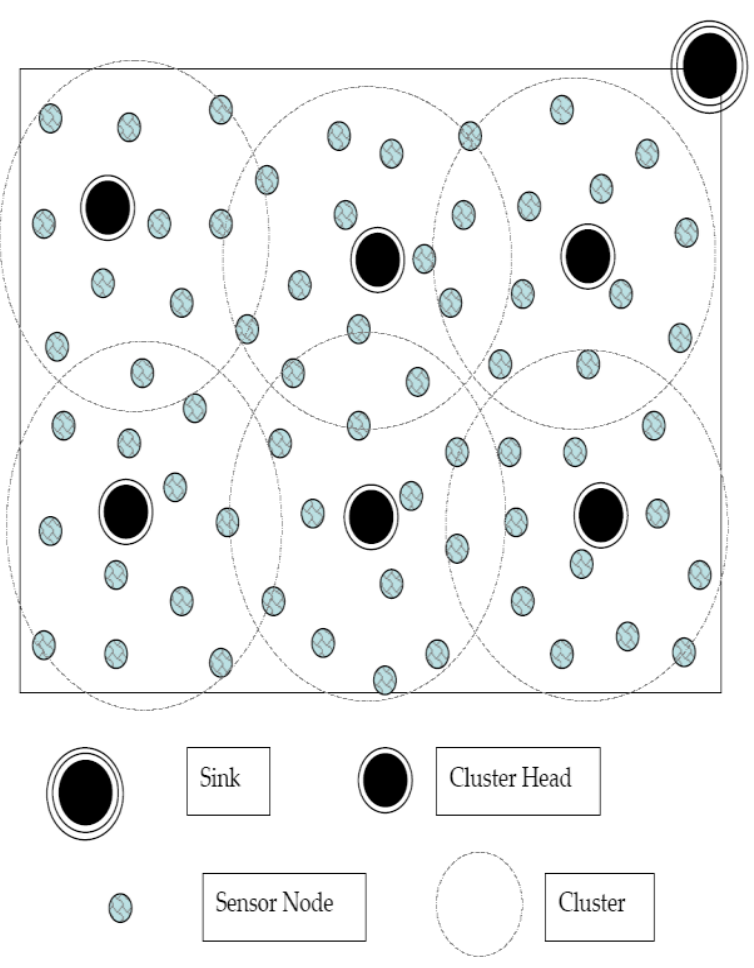

Fig 2: Architecture of a Hierarchical Network

1) All nods needs to transmit only to their $\mathrm{CH}$ rather than $\mathrm{CH}$, thus saving energy.

2) Only the $\mathrm{CH}$ are responsible to perform the task of data collecting and aggregation, thus again saving the energy of individual nodes.

3) In case HWSN's, CH have more resources and hence handle the additional energy requirements. 
4) The nodes in a cluster are adjacent and tend to have similar data. Since the local $\mathrm{CH}$ collect this data, aggregation is comparatively more effective.

\section{DESIGN AND IMPLEMENTATION ISSUES}

Implementing cluster based architecture requires a significant amount of work to be done. Clustering offers a wide range of advantages for a sensor network but still it has its own drawbacks, issues and challenges. In this section we outline several concrete design and implementation issues involved in the development of cluster based network architecture

\subsection{Node Mobility}

Most of the network architectures assume that nodes are stationary. But sometimes it is compulsory to support the mobility of base stations or CHs. Node mobility makes clustering a very challenging task since the node membership will dynamically change, forcing clusters to evolve over time.[4]

\subsection{Traffic Load}

Events that are monitored by a sensor network can either be continual or intermittent. Intermittent monitoring generates traffic in the network only when detecting the event of interest, whereas continual monitoring generates traffic at frequent intervals as they continually sense information. Since intermittent events requires only occasional sensing it does not reflect any change in the $\mathrm{CH}$, whereas intermittent events unevenly load $\mathrm{CHs}$ relative to the nodes in the cluster and a rotation of $\mathrm{CH}$ role may be required if the $\mathrm{CH}$ is randomly picked from the sensor population[4].

\subsection{Overlapping Clusters}

As stated earlier the cluster head $\mathrm{CH}$ may be predesigned by the network designer or elected by the sensors in the network. If the later one is opted there is a possibility that a member of one cluster may become the member under another $\mathrm{CH}$. This makes the overlapping clusters also to be considered in the design issues. It is therefore important to establish necessary mechanisms for detecting the existence of overlapped clusters and coordinating between clusters to avoid unfairness, starvation or deadlock during resource competition [7]

\subsection{Load Balancing}

Load balancing is one of the most pressing issues in sensor networks where $\mathrm{CHs}$ are picked from the available sensors. The member sensor nodes needs to be evenly distributed among the different $\mathrm{CHs}$ available which if fails will overload a particular $\mathrm{CH}$ leading to the failure of that head. So in such cases it is necessary to design equal sized clusters for a fair balancing.

\subsection{Dynamic Cluster Control}

It is necessary to configure a self configuring clustering mechanism with a sensor network. The clustering mechanisms are responsible for the formation of initial clusters which needs to adapt to its location. The clusters are formed based on several metrics like data accessibility, node capacity, network connectivity etc. One of the important design issues in clustering is the cluster head has to dynamically determine the membership of the nodes as the phenomenon moves. It has been noted in [7], that when the target is beyond the sensing range of the $\mathrm{CH}$, another round of head election is necessary to find a new $\mathrm{CH}$.

\subsection{Inter-cluster Coordination}

To achieve the desired goal CH's needed to communicate with each other. They might need to communicate for sharing of information and to achieve coordination. Further data gathered by one cluster can be requested by base station or other $\mathrm{CH}$ across the network. So the self configuring clustering mechanism should be capable of handling intercluster communication overheads.

\subsection{Data Aggregation}

The $\mathrm{CH}$ needs to perform the task of aggregating and transmitting the data from the nodes in the cluster to the $\mathrm{CH}$ and hence consumes more energy. So there should be a proper care taken while deciding the $\mathrm{CH}$. One way of conserving the energy of the CH's is by rotating the roles between different nodes, at periodic intervals. Another option is to have the powerful node that can handle the additional energy requirement, to act as the $\mathrm{CH}$.

\subsection{Fault Tolerance}

Fault tolerance is the ability to sustain sensor network functionalities without any interruption due to sensor node failures. Some sensor nodes may fail or be blocked due to lack of power, have physical damage or environmental interference. The failed node might be a $\mathrm{CH}$ or a member of the cluster. Such failures should not affect the overall task and performance of the sensor network. So it is therefore necessary to have a mechanism which will adapt to these types of failures.

\subsection{Scalability}

After the initial formation of clusters, the $\mathrm{CH}$ should be able to adapt to either increase or decrease in its cluster member's count. The member count of a cluster may change due to various factors. For example a cluster member may fail due to environmental threat. During this time the $\mathrm{CH}$ should adapt $\mathrm{t}$ a decrease in its member count. On the flip side, increase in the member count may also happen during circumstances like addition of new sensor nodes, failure of an existing $\mathrm{CH}$ etc. Similarly the sensor network itself should be capable of adapting to either increase or decrease in the number of clusters.

\subsection{Number of Clusters}

Total number of clusters or cluster count is another important design issue to be considered. It is necessary that the cluster count should be very optimal, which if fails leads to network complexity and management overhead. Formation of optimal number of clusters will make the network energy efficient.

\subsection{Cluster Formation Time}

The time taken by the network to form the initial cluster should be very minimal. Events including the choice of cluster count, selection of $\mathrm{CH}$, allotment of cluster members to a $\mathrm{CH}$ should be done with in minimum period of time.

\subsection{Single hop Vs Multi hops Network.}

Communication in clustering can be either single hop communication or multi hop communication. As the transmission energy varies directly with the square of distance therefore a multi -hop network is suitable for conserving energy [8]. But Multi hop network raises various design issues regarding to topology management and media access control. This is another issue to be considered. 


\subsection{Node Heterogeneity}

Some applications of sensor might require a diverse mixture of sensor nodes with different types and capabilities to be deployed. Data from different sensors can be generated at different rates; network can follow different data reporting models and can be subjected to different quality of service constraints. Such a heterogeneous model will make clustering a difficult one and making the job of $\mathrm{CH}$ as a tougher one. [8]

\subsection{Cluster Formation}

The cluster formation has to consider in to account several things like whether the cluster formation is centralized or localized, whether the number of clusters is assigned a priori or is it formed distributed etc. the cluster based routing protocols should address to such kind of design issues in sensor networks. To distribute energy load evenly among the sensors, each sensor in a cluster randomly becomes the cluster head. However the percentage of cluster heads in a network is pre assigned by the network designer.

\subsection{Self Configuration and \\ Reconfiguration}

One of the most important phases of cluster formation is the self - organization phase. The clusters should have the capability self configuring themselves. How fast the network self organize in did to functional unit is one of the important issues in wireless sensor network. To maximize the network life time the self organizing phase should be short and energy efficient. Another issue is Reconfiguration or Replenishment. Replenishment can be defined as the process of adding new fresh sensor nodes with full energy reserve to replace old and energy depleted sensors. Reconfiguration is the process of self - organization after the loss or addition of new sensor nodes.[9]

\section{SIMULATION}

We try to evaluate the performance of various clustering schemes via MATLAB simulation in this section. Various types of clustering schemes like clustering at the centre of the network (Static Cluster Head), Clustering at the perimeter of the network, Multiple Clustering by exploiting a mobile $\mathrm{CH}$ at the perimeter are been simulated and analyzed. We have shown how various clustering schemes affect the performance of the network. Our simulation setting is as follows. We placed set of sensor nodes in a two dimensional grid sensing area. First the $\mathrm{CH}$ or Sink is static and placed at the center of the sensing area. A data gathering tree is formed

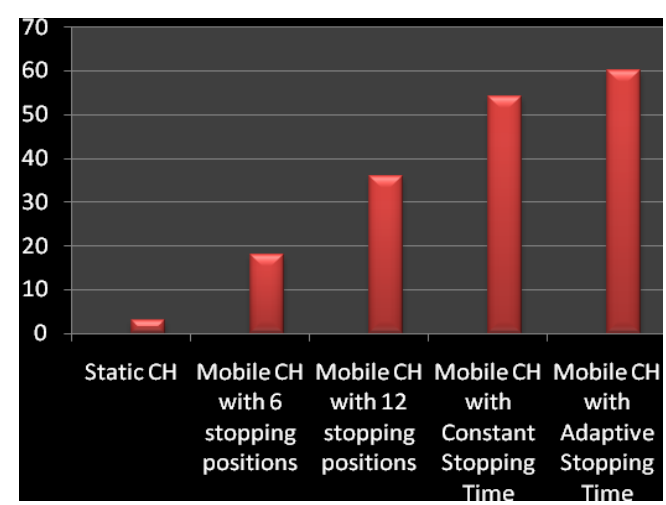

Fig 3: No of Rounds for the first node to Die

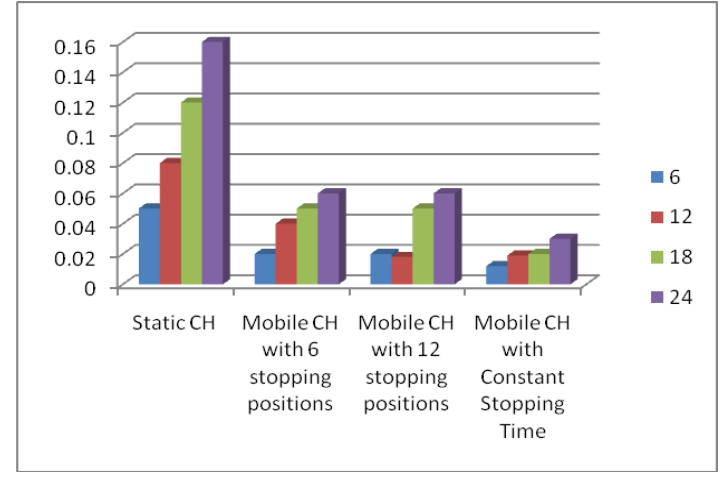

Fig 4: Approximate energy consumption at First Corona after $T$ rounds

To allow the sensor nodes around the $\mathrm{CH}$ to pass their sensed data to it. After a time period T, the residual energy of the one hop neighbors of the $\mathrm{CH}$ is considered and the number of rounds the $\mathrm{CH}$ is able to hold up before the first node to expire is analyzed. The energy consumption of the sensor nodes is also analyzed. Then the $\mathrm{CH}$ is changed as a mobile node and allowed to move along the perimeter of the sensing area. Different stopping positions for $\mathrm{CH}$ are also simulated and evaluated. Fig 3 shows the number of rounds for the first node to die (Here Network Lifetime is defined as the time until the first node dies) in different clustering schemes. Static $\mathrm{CH}$ holds very minimum number of rounds as it stays in the same position for a long time. Due to its static nature sensors around the $\mathrm{CH}$ will become the bottleneck nodes as they forward packets on behalf of other sensors in the network. So network lifetime is very less in the case of static $\mathrm{CH}$. Whereas Mobile $\mathrm{CH}$ shows significant improvement in network lifetime over static $\mathrm{CH}$. In Fig 4 approximate energy consumption of first corona sensors are analyzed. Energy consumption at first corona is more in Static $\mathrm{CH}$ when compared to mobile $\mathrm{CH}$. Then we have analyzed the energy consumption along the network for various clustering schemes. Fig 5 shows the energy consumption for static sink and it can be easily noticed that the energy consumption is more in the centre of the network. Whereas in mobile $\mathrm{CH}$ scheme (Fig 6,7) which moves along the perimeter of the network the energy consumption is scattered around the perimeter of the network. In random position $\mathrm{CH}$ (Fig 8) movement the energy consumption is fairly distributed along the network area.

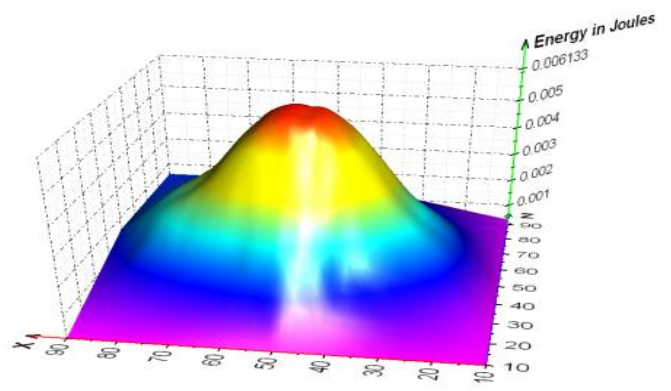

Fig 5: Static CH 


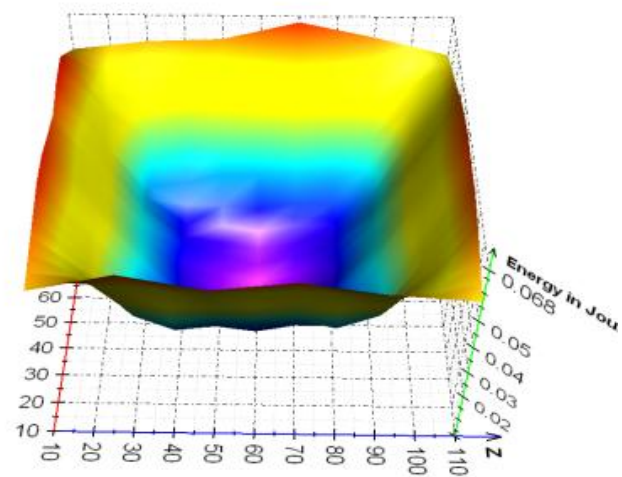

Fig 6 Mobile $\mathrm{CH}$ along the perimeter of Network (6 stopping Positions)

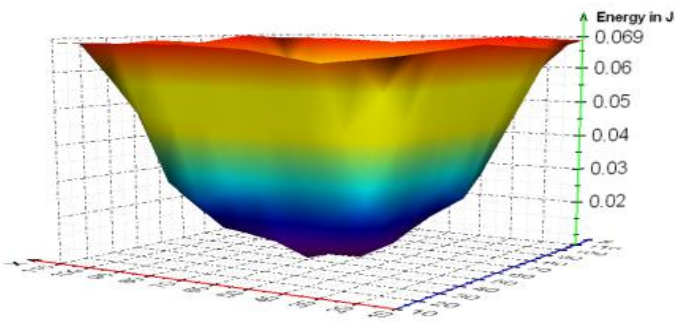

Fig 7: Mobile CH along the perimeter of Network (12 stopping Positions)

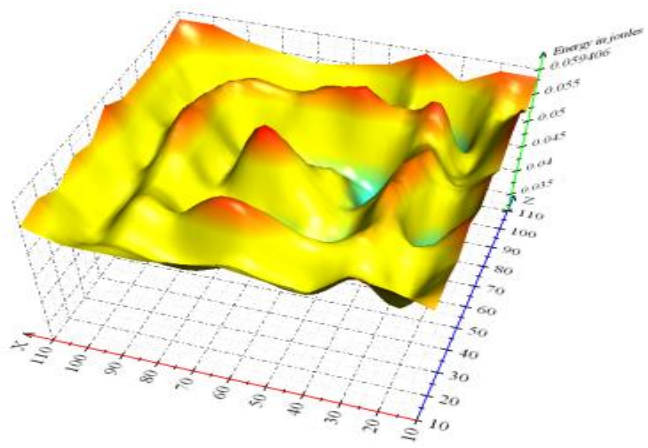

Fig 8: Mobile CH with Random Stopping Points

So we conclude that making the $\mathrm{CH}$ mobile and stopping it at various random points along the network shows a significant improvement on the sensor network lifetime.

\section{RESEARCH ISSUES IN CLUSTERING}

There are still many research issues that are open to the research community. Some of them are as follows

1. How could a Base station determine its closest cluster head?

2. How could a base station probe a certain region of the network through one of its nearby $\mathrm{CH}$.?

3. How to maintain the stability of the $\mathrm{CH}$ ?

4. How to overcome issues in inter cluster formation?

5. Formation of energy efficient clusters.

6. Handling of heterogeneous nodes.

7. Parameters for cluster formation.

8. Routing in clustering.

9. Localization in clustering.

How clustering can help to increase the network life time?

Thus we have discussed the various design and implementation issues and challenges in clustering for sensor networks. Although clustering provides a wide range of advantages for sensor network it does have some issues. So these issues have to be considered while designing a clustering protocol.

\section{REFERENCES}

[1] I.F Akylidiz, "Wireless Sensor Networks a survey", Computer Networks 38(2009)

[2] C-Y Chong, S.P Kumar, "Sensor Networks: Evolution, Opportunities, and Challenges", Proceedings of the IEEE $91(8)(2003)$

[3] D. Estrin, "Next century challenges: Scalable coordination in sensor Network", in: Proceedings of the Fifth International Conference on Mobile Computing and Networks (MobiCom ‘99)

[4] Ameer Ahmed Abbasi, Mohammed Younis "A Survey on clustering algorithms for Wireless Sensor Networks", Computer communications 30(2007), 2826-2841

[5] Ataul Bari, Arunita Jaekel and subir Bandyopadhyay, "Optimal placement and routing strategies for resilient two -tiered sensor networks": University of Windsor, school of computer science Canada. Wireless Communications and Mobile computing. 28 may 2008

[7] Yang Yu, Bhaskar Krishnamachari and Viktor K. Prasanna, Department of EE Systems, University of southern California "Issues in Designing Middleware for Wireless Networks"

[8] Nitaigour Premchand Mahalik "Sensor networks and configuration: fundamentals, standards, platforms, and Applications" Publisher: Springer. Publication Date: c2007

[9] J. Luo, J.-P. Hubaux, Joint mobility and routing for lifetime elongation in wireless sensor networks, IEEE INFOCOM, 2005.

[10] Y. Bi, L. Sun, J. Ma, N. Li, I.A. Khan, C. Chen, HUMS: An autonomous moving strategy for mobile sinks in data-gathering sensor networks, Eurasip, Journal on Wireless Communications and Networking,2007.

[11] M. Marta, M. Cardei, Improved sensor network lifetime with multiple mobile sinks, Pervasive and Mobile Computing (2009), doi:10.1016/j.pmcj.2009.01.001 
[12] Y. Bi, L. Sun, J. Ma, N. Li, I.A. Khan, C. Chen, HUMS: An autonomous moving strategy for mobile sinks in data-gathering sensor networks, in: Eurasip, 2007

[13] A. Chakrabarti, A. Sabharwal, B. Aazhang, Using predictable observer mobility for power efficient design of sensor networks, in: Proc. of the 2nd IEEE IPSN, 2003.

[14] Wei Wang, Vikram Srinivasan, and Kee-Chaing Chua, "Extending the Lifetime of Wireless Sensor Networks Through mobile relays", IEEE/ACM Transactions on Networking, Vol. 16, No. 5, pp.1108-1120,October 2008

[15] Wei Wang, Vikram Srinivasan, and Kee-Chaing Chua, "Using Mobile Relays to Prolog the Lifetime of Wireless Sensor Networks", in Proc. ACM MobiCom Aug.2005, pp 270-283.

[16] Banerjee, T.; Bin Xie; Jung Hyun Jun; Agrawal, D.P.," LIMOC: Enhancing the Lifetime of a Sensor Network with Mobile Cluster heads", Vehicular Technology Conference, 2007. VTC-2007 Fall. 2007 IEEE 66th Volume, Issue, Sept. 30 2007-Oct. 32007 Page(s):133 137 Digital Object Identifier $\quad 0.1109$ /VETECF.2007.43

[17] I. F. Akyildiz, W. Su, Y. Sankarasubramaniam, and E. Cayirci, "Wireless sensor networks: A survey", IEEE Communications Magazine, vol 40, no 8, pp.102-114, Aug 2002.
[18] J. Li, P. Mohapatra, An analytical model for the energy hole problem in many-to-one sensor networks, in: Vehicular Technology Conference, 2005.

[19] S. Olariu, I. Stojmenovic, Design guidelines for maximizing lifetime and avoiding energy holes in sensor networks with uniform distribution and uniform reporting, in: IEEE INFOCOM, 2006.

\section{AUTHOR'S PROFILE}

K.Pradeepa received her Bachelors degree in Computer Science and Masters in Computer Applications from Bhrathiar Universty, Coimbatore, India. She is currently working as Assistant Professor in the Department of Computer Applications at Sri Krishna College of Engineering and Technology, Coimbatore. Her areas of interest include Wireless Sensor Networks and Analysis of Algorithms.

W.Regis Anne received her Bachelors degree in Physics and Masters in Computer Applications from Anna University. She is currently working as Assistant Professor in the Department of Computer Applications at Sri Krishna College of Engineering and Technology, Coimbatore, India. Her areas of interest include Wireless Sensor Networks.

Dr S.Duraisamy received his Doctorate in Computer Science and Engineering from Alagappa Universty, Karaikudi, India. $\mathrm{He}$ is currently working as the Head and Professor of the Department of Computer Applications at Sri Krishna College of Engineering and Technology, Coimbatore, India. His areas of interest include Software Engineering, Object Oriented Concepts and Wireless Sensor Networks. 